\title{
Observation of Astrophysical Neutrinos in Four Years of IceCube Data
}

\author{
The IceCube Collaboration \\ $\dagger$ http://icecube.wisc.edu/collaboration/authors/icrc15_icecube \\ E-mail: ckopper@icecube.wisc.edu, will.giang@icecube.wisc.edu, \\ naoko@drexel .edu
}

The spectrum of cosmic rays includes the most energetic particles ever observed. The mechanism of their acceleration and their sources are, however, still mostly unknown. Observing astrophysical neutrinos can help solve this problem. Because neutrinos are produced in hadronic interactions and are neither absorbed nor deflected, they will point directly back to their source. This contribution will cover continued studies of the high-energy astrophysical neutrino flux observed at the IceCube neutrino observatory, extending them from three to four years of data with a focus on energies above $60 \mathrm{TeV}$. The spectrum and spatial clustering of the observed neutrinos will be discussed.

Corresponding authors: C. Kopper*1, W. Giang ${ }^{2}$, N. Kurahashi ${ }^{2}$

1 Department of Physics, CCIS 4-181, University of Alberta, Edmonton, Alberta T6G 2E1, Canada

2 Department of Physics, Drexel University, 3141 Chestnut Street, Philadelphia, PA 19104, USA

The 34th International Cosmic Ray Conference,

30 July- 6 August, 2015

The Hague, The Netherlands

${ }^{*}$ Speaker. 


\section{Introduction}

Observation of high-energy neutrinos provides insight into the problem of the origin and acceleration mechanism of high-energy cosmic rays. Cosmic ray protons and nuclei interacting with gas and photons present in the environment of sources and in the interstellar space produce neutrinos through decay of charged pions and kaons. These neutrinos have energies proportional to the cosmic rays that produced them and point back to their source since neutrinos are neither affected by magnetic fields nor absorbed by matter opaque to radiation. Large-volume Cherenkov detectors like IceCube [1] observe these neutrinos when they interact by measuring the light produced by secondary leptons and hadronic showers.

Here we present an update to the IceCube high-energy search for events with interaction vertices inside the detector fiducial volume. This search, previously performed on two [2] and three [3] years of detector data, led to the discovery of an astrophysical neutrino flux above atmospheric backgrounds [3]. This update extends the data-taking period by one more year to four years from 2010 to 2014 for a total livetime of 1347 days.

\section{Event Selection}

Neutrinos in IceCube are detected by observing the Cherenkov light in the glacial ice from secondary particles created by the interaction of high-energy neutrinos. We observe two main event classes: track-like events from charged-current interactions of muon neutrinos (and from a minority of tau neutrino interactions) and shower-like events from all other interactions (neutralcurrent interactions and charged-current interactions of electron and tau neutrinos). Note that tau neutrino interactions at the highest energies (at the PeV-level) can lead to different event shapes in rare cases, but these are not considered here. We determine the event direction and deposited energy in the detector based on the time sequence and total recorded light. Energy is reconstructed as electromagnetic-equivalent energy. Shower-like events have a typical directional resolution of around $15^{\circ}$, mostly dominated by the uncertainty in the modelling of the glacial ice, whereas tracklike events have resolutions of much better than $1^{\circ}$.

In the analysis presented here, signal events are selected by requiring the neutrino interaction vertex to be located inside the IceCube detector volume. We achieve this by means of a simple anticoincidence veto method [2], requiring that fewer than 3 of the first 250 detected photoelectrons (p.e.) be on the outer detector boundary. In order to ensure a large enough number of photons are detected, we also require at least 6000 p.e. in total charge for each event, corresponding to a soft threshold of about $30 \mathrm{TeV}$ in deposited energy in the detector.

\section{Atmospheric Backgrounds}

Backgrounds to astrophysical neutrinos are entirely due to cosmic ray air showers. Muons produced in these showers, mainly from $\pi$ and $\mathrm{K}$ decays enter the detector from above. Due to the stochasticity of the muon energy loss, in very rare cases muons can pass through the outer veto layer undetected and appear as starting events, especially close to the charge threshold of 6000 p.e. We use a data-driven method to estimate this background by tagging such muons in one layer of 
the detector and then use a second layer, equivalent to our veto to estimate their passing probability. From this, we estimate a total atmospheric muon background of $12.6 \pm 5.1$ in four years of data.

The same cosmic ray air showers also produce neutrinos from $\pi$ and $K$ decays. The spectrum of these $v_{\mu}$-dominated atmospheric neutrinos is typically one power steeper than the original cosmic ray spectrum. This is due to the increasing lifetime of the parent mesons, making it more and more likely for it to interact before decaying. At energies above around $100 \mathrm{TeV}$, an analogous flux of muons and neutrinos from charmed mesons is expected to dominate. The shorter lifetime of these particles allows them to avoid interactions before their decay, leading to a harder spectral slope of this component. Until now, this "prompt" component has not yet been observed, but limits from data have been placed by previous IceCube analyses [4, 5]. For purposes of background estimation, we use a limit set by an analysis of incoming muons from muon neutrinos in the 59-string configuration of IceCube [4]. More recent and somewhat more stringent limits are available from IceCube data [5], however we chose to continue to use the same limit as before because it was chosen during the blind definition of this analysis update. The total expected background from atmospheric neutrinos in four years of data including this limit is $9.0_{-2.2}^{+8.0}$.

Note that some of the down-going atmospheric neutrino background will also be vetoed because of accompanying muons from the same air shower. This reduces the background from atmospheric neutrinos in the Southern Sky. This analysis uses the updated description of this reduction described in reference [5].

\section{Diffuse Flux Fit}

In the full 1347-day sample, we detect 54 events (Fig. 1); 17 of them are observed in the fourth year (see table 1). One of the events observed in the third year (event \#32) was produced by a coincident pair of background muons from unrelated cosmic ray air showers and has been excluded from the analysis. We note that none of the events in the fourth year have energies above $1 \mathrm{PeV}$. We do, however, observe one very high-energy track (event \#45) with an almost vertical direction starting in the top part of the detector and depositing more than $400 \mathrm{TeV}$ in the detector before leaving it at the bottom. If this event was a muon produced from a cosmic ray interacting in the atmosphere, the air shower secondaries would likely have been observed by the surface IceTop array (see [6] in these proceedings). Distributions of the events compared to background and best-fit signal expectations as functions of deposited charge, deposited energy and declination can be found in figures 2, 3 and 4, respectively.

In order to describe the data, we perform a likelihood fit of all components (atmospheric muons, atmospheric neutrinos from $\pi / \mathrm{K}$ decay, atmospheric neutrinos from charm decay and an astrophysical flux assuming a 1:1:1 flavor ratio). As in [3], the fit is performed including all events with $60 \mathrm{TeV}<E_{\text {dep }}<3 \mathrm{PeV}$. The only change in fitting procedure from previous analyses is the addition of a prior distribution on the charm background component in line with the limit on this component from the previous IceCube 59-string analysis on incoming muon neutrinos [4]. We fit an unbroken power-law spectrum, both with a fixed index of $E^{-2}$ and with a variable index $E^{-\gamma}$. For the fixed spectral index, we obtain a normalization of $E^{2} \Phi(E)=0.84 \pm 0.3 \times 10^{-8} \mathrm{GeVcm}^{-2} \mathrm{~s}^{-1} \mathrm{sr}^{-1}$, compatible with previous results. Note, however, that this spectral assumption is not strongly favored by the data, especially with the addition of several new lower-energy events to the sample in 


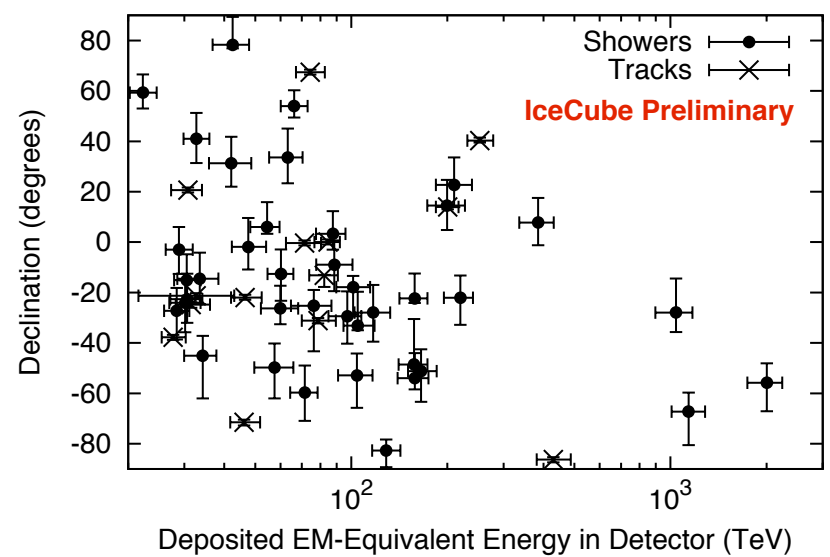

Figure 1: Arrival angles and electromagnetic-equivalent deposited energies of the events. Track-like events are indicated with crosses whereas shower-like events are shown as filled circles. The error bars show $68 \%$ confidence intervals including statistical and systematic errors. Deposited energy as shown here is always a lower limit on the primary neutrino energy.

\begin{tabular}{c|c|c|c|c|c|c} 
ID & $E_{\text {dep }}(\mathrm{TeV})$ & Time (MJD) & Decl. (deg.) & R.A. (deg.) & Ang. Err. (deg.) & Topology \\
\hline 38 & $200.5_{-16.4}^{+16.4}$ & 56470.11038 & 13.98 & 93.34 & $\lesssim 1.2$ & Track \\
39 & $101.3_{-11.6}^{+13.3}$ & 56480.66179 & -17.90 & 106.17 & 14.2 & Shower \\
40 & $157.3_{-16.7}^{+15.9}$ & 56501.16410 & -48.53 & 143.92 & 11.7 & Shower \\
41 & $87.6_{-10.0}^{+8.4}$ & 56603.11169 & 3.28 & 66.09 & 11.1 & Shower \\
42 & $76.3_{-11.3}^{+10.3}$ & 56613.25669 & -25.28 & 42.54 & 20.7 & Shower \\
43 & $46.5_{-4.5}^{+5.9}$ & 56628.56885 & -21.98 & 206.63 & $\lesssim 1.3$ & Track \\
44 & $84.6_{-7.9}^{+7.4}$ & 56671.87788 & 0.04 & 336.71 & $\lesssim 1.2$ & Track \\
45 & $429.9_{-49.1}^{+57.4}$ & 56679.20447 & -86.25 & 218.96 & $\lesssim 1.2$ & Track \\
46 & $158.0_{-16.6}^{+15.3}$ & 56688.07029 & -22.35 & 150.47 & 7.6 & Shower \\
47 & $74.3_{-7.2}^{+8.3}$ & 56704.60011 & 67.38 & 209.36 & $\lesssim 1.2$ & Track \\
48 & $104.7_{-10.2}^{+13.5}$ & 56705.94199 & -33.15 & 213.05 & 8.1 & Shower \\
49 & $59.9_{-7.9}^{+8.3}$ & 56722.40836 & -26.28 & 203.20 & 21.8 & Shower \\
50 & $22.2_{-2.0}^{+2.3}$ & 56737.20047 & 59.30 & 168.61 & 8.2 & Shower \\
51 & $66.2_{-6.1}^{+6.7}$ & 56759.21596 & 53.96 & 88.61 & 6.5 & Shower \\
52 & $158.1_{-18.4}^{+16.3}$ & 56763.54481 & -53.96 & 252.84 & 7.8 & Shower \\
53 & $27.6_{-2.2}^{+2.6}$ & 56767.06630 & -37.73 & 239.02 & $\lesssim 1.2$ & Track \\
54 & $54.5_{-6.3}^{+5.1}$ & 56769.02960 & 5.98 & 170.51 & 11.6 & Shower
\end{tabular}

Table 1: Properties of the events observed in the fourth year. A list of events \#1-\#37 can be found in [3]. The $E_{\text {dep }}$ column shows the electromagnetic-equivalent deposited energy of each event. "Ang. Err." shows the median angular error including systematic uncertainties. 


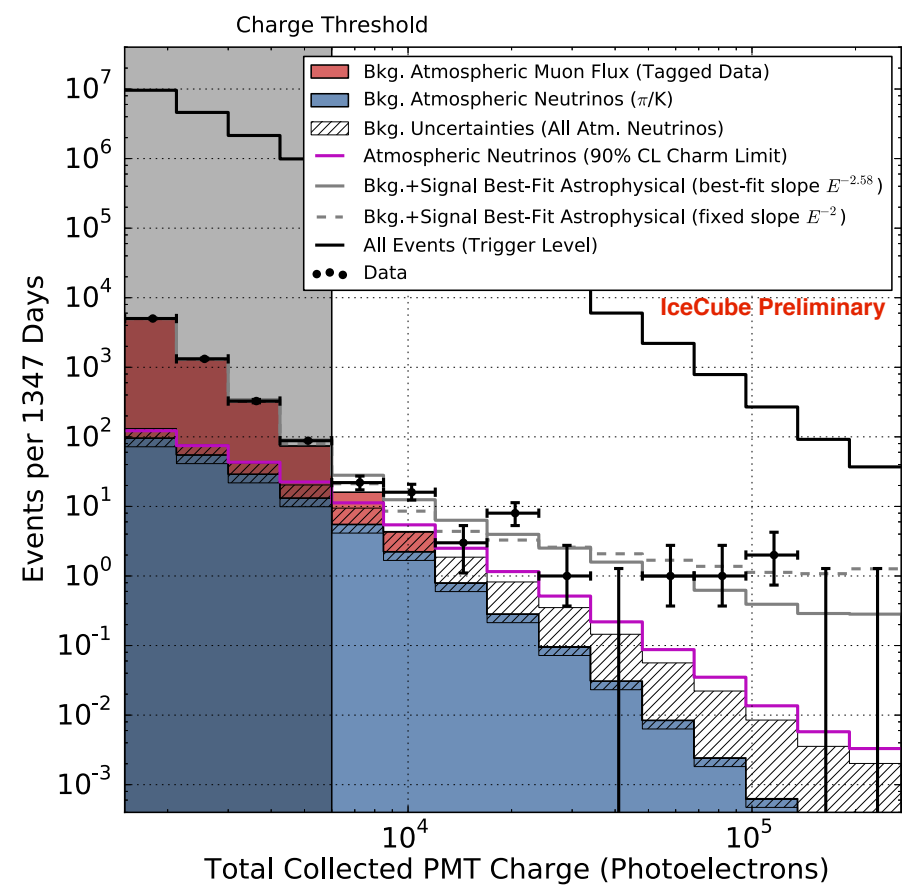

Figure 2: Distribution of deposited PMT charges of the events. Atmospheric muon backgrounds (estimated from data) are shown in red. Due to the incoming track veto, these backgrounds fall much faster than the overall background at trigger level (black line). The data events in the unshaded region at charges greater than 6000 p.e. are the events reported in this work. Atmospheric neutrino backgrounds are shown in blue with $1 \sigma$ uncertainties on the prediction shown as a hatched band. For scale, the $90 \%$ CL upper bound on the charm component of atmospheric neutrinos is shown as a magenta line. The best-fit astrophysical spectra (assuming an unbroken power-law model) are shown in gray. The dashed line shows a fixed-index spectrum of $E^{-2}$, whereas the solid line shows a spectrum with a best-fit spectral index.

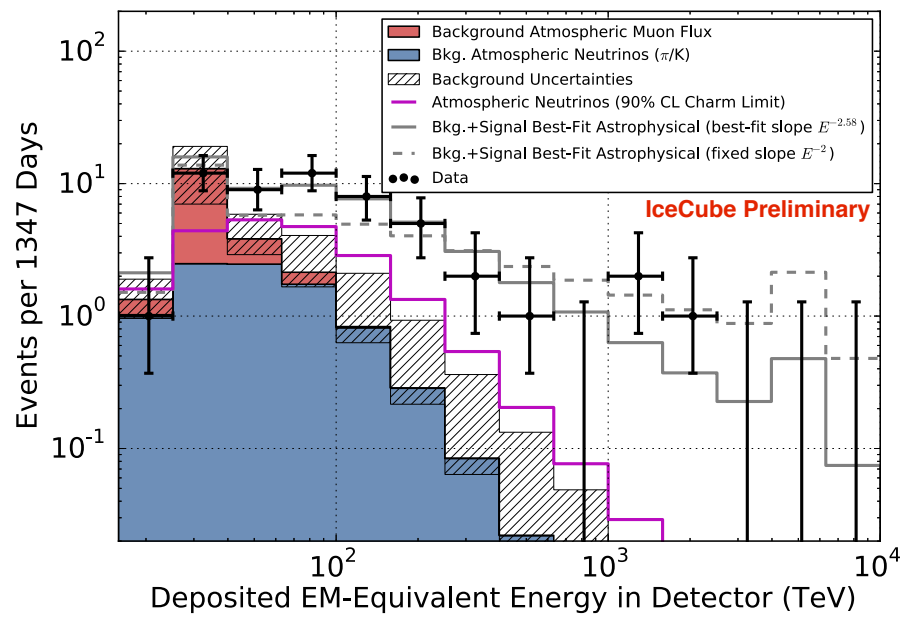

Figure 3: Deposited energies of the observed events with predictions. Colors as in Fig. 2. 


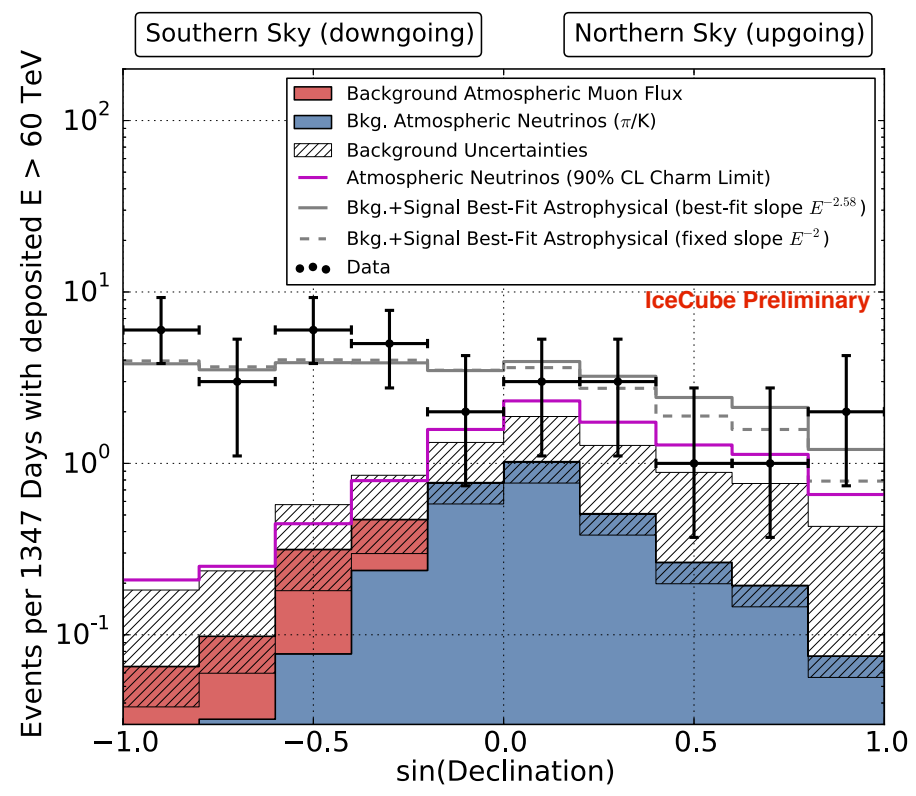

Figure 4: Arrival angles of events with $E_{\mathrm{dep}}>60 \mathrm{TeV}$ compared to predictions. Colors as in Fig. 2.

the fourth year of data (see gray dashed line in Fig. 3). The variable spectral index fit results in a best-fit spectral index of $-2.58 \pm 0.25$, softer than the corresponding best-fit index of $-2.3 \pm 0.3$ obtained with three years of data. The new fit is compatible with the 3-year result within errors (see Fig. 5); however, the lack of PeV-energy events in the fourth year of data in combination with the comparatively high yield of events in that year has resulted in a much steeper spectral fit.

Fig. 6 shows a fit of the spectrum using a more general model, parameterizing the astrophysical flux as a piecewise function of neutrino energy instead of an unbroken single power law. The new dataset presented here is also used in a global fit of several IceCube analyses, presented in these proceedings [7].

\section{Spatial Clustering}

A maximum-likelihood clustering method [3] was used to look for any neutrino point source in the sample. The test statistic (TS) was defined as the logarithm of the ratio between the maximal likelihood including a point source component and the likelihood for the isotropic null hypothesis. The significance of our observed TS was determined by comparing to maps scrambled in right ascension. As before, the analysis was run twice, once with all events and once with only shower-like events in the sample. We removed events \#32 (two coincident muons from unrelated air showers) and \#28 (event with sub-threshold hits in the IceTop array) for purposes of all clustering analyses. This test (see Fig. 7) did not yield significant evidence of clustering with p-values of $44 \%$ and $58 \%$ for the shower-only and the all-events tests, respectively.

We also performed a galactic plane clustering test using a fixed width of $2.5^{\circ}$ around the plane (p-value 7\%) and using a variable-width scan (p-value 2.5\%). All above p-values are corrected for trials. 


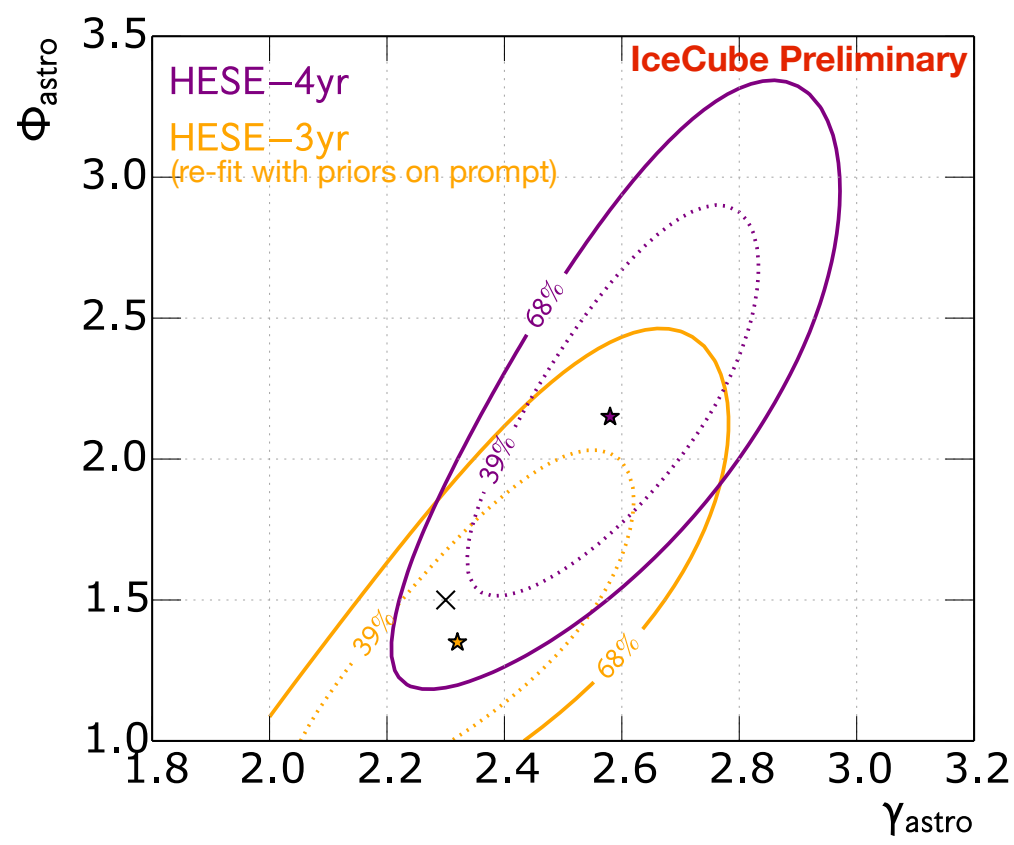

Figure 5: Contour plot of the best-fit astrophysical spectral index $\gamma_{\text {astro }}$ vs. best-fit normalization at

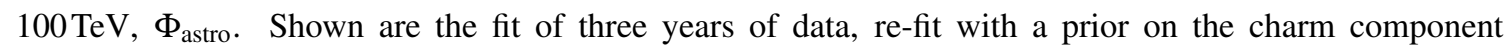
in yellow ("HESE-3year"). The best-fit point is marked with a yellow star. The previous fit as shown in [3] is marked with a black " $x$ " (this fit used an unconstrained charm component). The fit of all four years of data using the same method is shown in purple ("HESE-4year") with a best-fit spectral index of $E^{-2.58 \pm 0.25}$, compatible with the 3-year result (although, note that the data used for the 3-year result is a subset of the 4-year result and thus the two are not independent.) The best-fit power law is $E^{2} \phi(E)=2.2 \pm 0.7 \times 10^{-8}(E / 100 \mathrm{TeV})^{-0.58} \mathrm{GeVcm}^{-2} \mathrm{~s}^{-1} \mathrm{sr}^{-1}$.

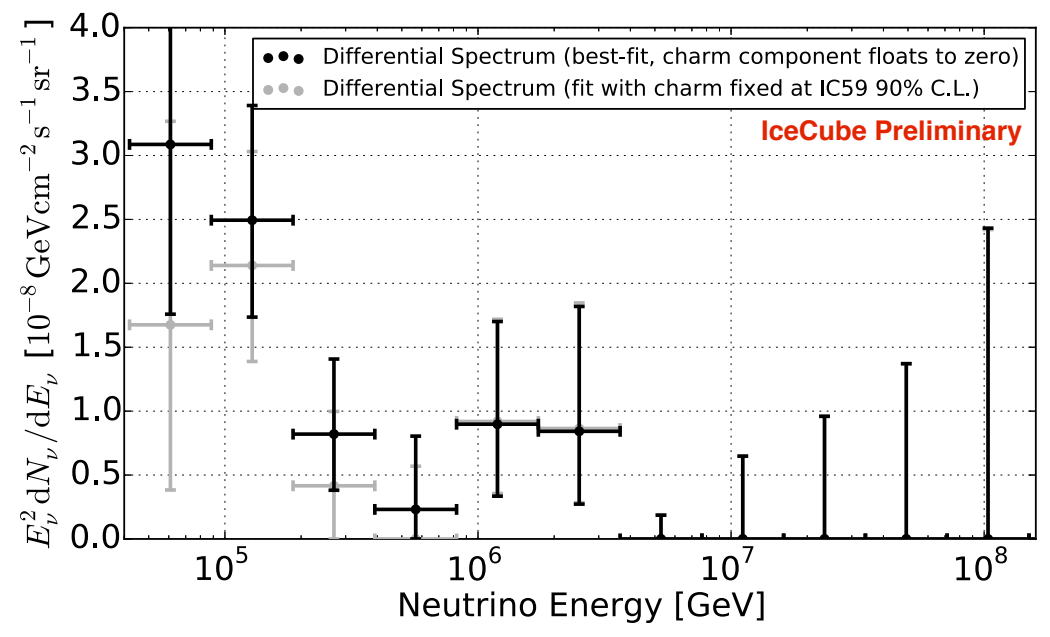

Figure 6: Astrophysical neutrino flux (combined neutrino and anti-neutrino) as a function of energy extracted from a combined likelihood fit of all background components and several pieces of $E^{-2}$ components in neutrino energy. Error bars indicate the $2 \Delta \mathscr{L}= \pm 1$ contours of the flux in each energy bin. An increase in the charm atmospheric background to the level of the $90 \%$ CL limit from the northern hemisphere $v_{\mu}$ spectrum [4] would reduce the inferred astrophysical flux at low energies to the level shown for comparison in light gray. 


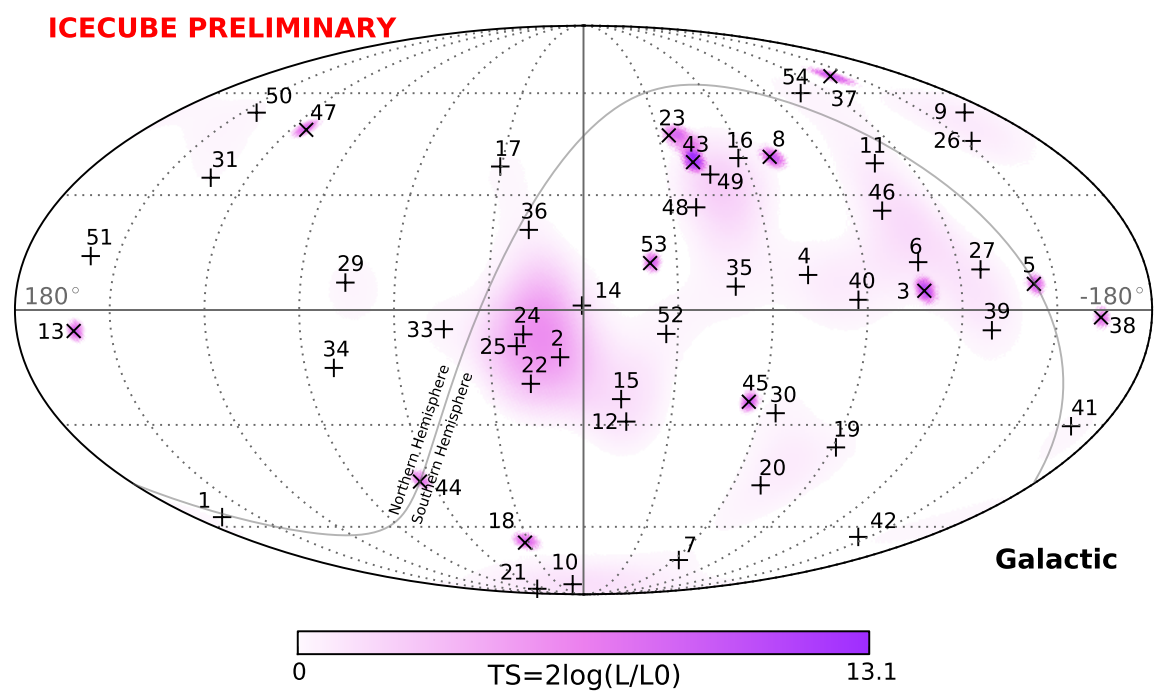

Figure 7: Arrival directions of the events in galactic coordinates. Shower-like events are marked with + and those containing tracks with $\times$. Colors show the test statistics (TS) for the point-source clustering test at each location. No significant clustering was found.

\section{Future Plans}

Other searches in IceCube have managed to reduce the energy threshold for a selection of starting events even further in order to be better able to describe the observed flux and its properties [5], but at this time they have only been applied to the first two years of data used for this study. We will continue these lower-threshold searches and will extend them to the full set of data collected by IceCube. Because of its simplicity and its robustness with respect to systematics when compared to more detailed searches, the search presented here is well suited towards triggering and providing input for follow-up observations by other experiments. In the future, we thus plan to continue this analysis in a more automated manner in order to update the current results with more statistics and to produce alerts as an input for multi-messenger efforts.

\section{References}

[1] R. Abbasi et al., Nucl. Instrum. Meth. A601 (2009) 294

[2] M.G. Aartsen et al., Science 342, 1242856 (2013)

[3] R. Abbasi et al., PRL 111 (2013) 021103

[4] M. G. Aartsen et al., PRD89 (2014) 062007

[5] M. G. Aartsen et al., PRD91 (2015) 022001

[6] IceCube Coll., PoS(ICRC2015)1086, these proceedings

[7] IceCube Coll., PoS(ICRC2015)1066, these proceedings 